\title{
Dominio de Arquitectura Empresarial, Armonizando la Simulación de Procesos y la Metodología de Desarrollo de Arquitectura (ADM)
}

\author{
Jorge E. Puerta-Ramírez y Jaime A. Giraldo-García \\ Universidad Nacional de Colombia, Facultad de Ingeniería y Arquitectura, Departamento de Ingeniería \\ Industrial, Campus La Nubia Bloque Q piso 2, Manizales - Colombia. \\ (e-mail: jepuertar@unal.edu.co, jaiagiraldog@unal.edu.co)
}

Recibido Abr. 4, 2016; Aceptado May. 26, 2016; Versión final Jun. 16, 2016, Publicado Dic. 2016

\begin{abstract}
Resumen
El objetivo de este trabajo es especificar un dominio de arquitectura empresarial, armonizando la simulación de procesos con la metodología de Desarrollo de Arquitectura (ADM, Architecture Development Method), en uno de los macro procesos de la Contraloría General de la República de Colombia. Se identificó el proceso de control fiscal micro, como clave para el cumplimiento de la misión de la organización. Se utilizó una herramienta de modelado y simulación basada en el estándar BPSim (Business Process Simulation) para detallar las fases del ciclo ADM. El modelado y la simulación mostraron una organización lógica del proceso estudiado y determinaron las dimensiones para limitar el alcance de la actividad de arquitectura. Con base en los resultados se concluye que la construcción de una arquitectura empresarial permite dimensionar de una manera más apropiada las necesidades de la organización.
\end{abstract}

Palabras clave: arquitectura empresarial, metodología ADM, modelamiento y simulación de procesos, estándar BPSim

\section{Enterprise Architecture Domain, Harmonizing Process Simulation and Architecture Development Methodology (ADM)}

\begin{abstract}
The aim of this study is to specify a domain of enterprise architecture, harmonizing process simulation methodology with ADM (Architecture Development Method), one of the macro processes of the Comptroller General of the Republic of Colombia. The micro fiscal control process was identified as the key element to fulfill the mission of the organization. A modeling and simulation tool based on BPSim standard (Business Process Simulation) to detail the stages of the ADM cycle was used. Modeling and simulation showed a logical organization of the study process and determined the dimensions to limit the scope of activity architecture. Based on the results it is concluded that the construction of enterprise architecture allows dimensioning the needs of the organization in a more appropriate way.
\end{abstract}




\section{INTRODUCCIÓN}

La CGR (Contraloría General de la República) tiene gran responsabilidad en el entramado institucional para contrarrestar la corrupción en Colombia, ya que la Constitución Política de 1991, la instituye como el máximo órgano de control fiscal para ejercer, en representación de la comunidad, la vigilancia de la gestión fiscal y de los particulares o entidades que manejan fondos o bienes de la Nación, en procura del buen uso de los recursos públicos y de contribuir a la modernización del estado mediante acciones de mejoramiento continuo. (CGR, 2011). Bajo estas premisas la CGR tiene una serie de retos políticos, jurídicos y de gestión, siendo éste último un factor importante a la hora de demostrar su eficiencia y efectividad en el control fiscal micro, que como lo afirma De Velasco (2004), lleva implícito los conceptos de objetivo y de mejora, los cuales aportan una sólida ventaja competitiva propia y sostenible en el tiempo. Cosa que no debe ser ajena a la CGR, si consideramos lo que dice González (2009), en cuanto a la necesidad de entender la entidad pública bajo el concepto de empresa, cuya gestión debe desarrollarse con la incorporación de las funciones del ciclo del proceso administrativo, establecidas en la teoría neoclásica de la administración en combinación con el marco jurídico aplicable a las entidades públicas.

Este reto de gestión de acuerdo con manifestaciones en los medios nacionales de comunicación por parte de representantes del gobierno nacional, el congreso de la república, los gremios, las organizaciones no gubernamentales, así como de académicos y analistas políticos, y en las propias palabras del Contralor General Edgardo Maya Villazón, está lejos de alcanzarse por parte de la CGR. Las manifestaciones mencionadas, ponen en tela de juicio la gestión de la CGR y han generado rumores acerca de una posible reestructuración, de transformar la contraloría en un tribunal de cuentas e incluso de la privatización del control fiscal en Colombia. Dichas afirmaciones se sustentan más en lo político y coyuntural del problema de la corrupción, que en estudios serios que demuestren cuales son las causas de la falta de eficiencia y efectividad de la entidad. En este mismo sentido, la AGR (Auditoría General de la República, 2013), ya ha advertido que la falta de calidad en el ejercicio del control fiscal, está determinada por fallas estructurales en sus procesos a la hora de alcanzar los estándares mínimos de pertinencia, oportunidad, eficiencia y eficacia con que las contralorías planifican, ejecutan y supervisan las auditorias.

Ante esto autores como Geyer y Fourie (2015), argumentan que las organizaciones deben definir formalmente y documentar sus procesos de negocio con el fin de entenderlos y así permitir un mejoramiento continuo de su gestión. Es por este motivo que se buscó determinar las características de los flujos de trabajo del proceso auditor. Como medio para determinar dichas características y para la comprensión de los procesos de la CGR, se utilizó el concepto de arquitectura empresarial, específicamente el dominio de arquitectura de negocio que como lo definen Serna, Salazar y Cortés (2010), se encarga entre otros, de la descripción de la estructura organizacional y de los procesos de negocio a través del modelamiento, que como manifiestan Bolaños y López (2012), es una técnica que permite captar las características principales del proceso, identificando su flujo y sus componentes para poder así comunicarlo con otros procesos, comprender las relaciones entre sus partes y conocer sus actores, responsables y demás artefactos que intervienen en su ejecución. Por otro lado y con el fin de analizar el modelo de procesos desarrollado, se utilizó la simulación como herramienta para representar el funcionamiento del mismo, que como afirma Villanueva (2008), es un procedimiento que nos permite analizar el comportamiento del sistema bajo diferentes circunstancias, el análisis de todos los cambios posibles y sus consecuencias, es decir, reemplazando variables y analizando el desempeño de los procesos hasta obtener la mejor alternativa.

A partir de los planteamientos anteriores, se realizó el modelado y simulación utilizando una herramienta tecnológica BPSim (Business Process Simulación) bajo los estándares BPMN (notación gráfica creada para proveer un lenguaje unificado de acepción mundial) del OMG (Object Management Group 2011) y XPDL (formato estándar para el intercambio de definiciones de procesos de negocio entre diferentes productos) definido por la WfMC (Workflow Management Coalition 2005) que permiten visualizar el impacto de cambios propuestos a los procesos empresariales antes de su implementación en el mundo real, como se afirma en Bizagi (2014) o en un proceso identificado como clave para el cumplimiento de la misión de la CGR.

\section{INVESTIGACIÓN PRELIMINAR}

La ausencia de estudios donde se identifiquen claramente las causas de la falta de eficiencia y efectividad de la CGR, conllevó a realizar una investigación preliminar que permitiera determinar la naturaleza y el alcance del problema. Dicha investigación inicio con una serie de entrevistas a funcionarios expertos en el tema y a partir de su análisis, se realizó una encuesta piloto. Las entrevistas se realizaron de forma semiestructurada buscando comparar las respuestas obtenidas, pero a su vez también permitiendo de alguna manera profundizar en aspectos relevantes para la investigación. La selección de los entrevistados se realizó de acuerdo con su disponibilidad, experiencia y rol desempeñado dentro del macro proceso de control fiscal micro, teniendo en cuenta una mínima representatividad estadística. Se entrevistó a un grupo 
de cinco (5) funcionarios de la Gerencia Departamental Risaralda de la CGR que desempeñan diferentes roles dentro del proceso.

Es importante aclarar que las entrevistas iniciaban indagando por las causas que generan ineficiencia e inefectividad en el ejercicio del control fiscal de la CGR y a partir de las respuestas dadas por los funcionarios, fueron emergiendo nuevas preguntas que permitieron profundizar en el tema. Al analizar y confrontar la información resultante de las entrevistas, se agruparon las causas en categorías para su posterior análisis (tabla 1).

Tabla 1. Análisis de causas por categorías

\begin{tabular}{|c|c|c|}
\hline Categoría & Causa & Síntesis de la explicación de los entrevistados \\
\hline 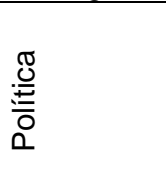 & $\begin{array}{l}\text { Modelo de } \\
\text { control fiscal }\end{array}$ & $\begin{array}{l}\text { A pesar de las bondades del modelo establecido en la Constitución Política de } \\
1991 \text {, se detectan muchas deficiencias que según los entrevistados provienen } \\
\text { desde el mismo congreso de la república cuando elige al Contralor General y } \\
\text { que trae consigo el clientelismo, restándole independencia al ejercicio del } \\
\text { control fiscal en Colombia. }\end{array}$ \\
\hline$\frac{.00}{\frac{.0}{0}}$ & $\begin{array}{l}\text { Enfoque en la } \\
\text { legalidad }\end{array}$ & $\begin{array}{l}\text { Al realizar el proceso auditor, el excesivo enfoque en la revisión del } \\
\text { cumplimiento de la normatividad, no permite la correcta valoración de la } \\
\text { inversión y el impacto que tiene está sobre la comunidad. }\end{array}$ \\
\hline \multirow{2}{*}{ 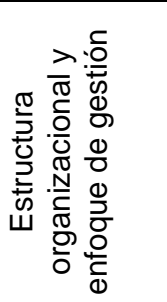 } & $\begin{array}{l}\text { Burocracia en } \\
\text { la entidad }\end{array}$ & $\begin{array}{l}\text { Como principal rasgo de una burocracia instituida, en la mayoría de ocasiones } \\
\text { priman los intereses propios de las altas directivas, sobre los argumentos } \\
\text { técnicos de los funcionarios de la entidad. }\end{array}$ \\
\hline & $\begin{array}{l}\text { Situación } \\
\text { laboral }\end{array}$ & $\begin{array}{l}\text { Debido a lo estricta que es la estructura organizacional, los funcionarios } \\
\text { sienten que no pueden influir sobre su trabajo, ni apoyarse o apoyar a un } \\
\text { compañero aprovechando sus capacidades profesionales. La rigidez y } \\
\text { verticalidad en la comunicación no posibilita que se alcance un mejoramiento } \\
\text { continuo en los procesos de la entidad. }\end{array}$ \\
\hline
\end{tabular}

Las primeras dos categorías se descartan por estar fuera del alcance del estudio y la investigación se concentra en la tercera, ya que en ésta coincidieron todos los entrevistados y fue donde se alcanzó un mayor grado de profundización en sus causas. A partir de este análisis se adoptó una encuesta propuesta y validada por el DAFP (Departamento Administrativo de la Función Pública de Colombia), la cual tiene como principal objetivo el diagnóstico de la estructura organizacional y el enfoque de gestión. Debido a la buena disposición y al receso del proceso auditor, la encuesta fue aplicada con una muestra censal a cuarenta (40) funcionarios pertenecientes al grupo de vigilancia fiscal de la Gerencia Departamental Risaralda de la CGR.

Tabla 2. Resultados de la encuesta

\begin{tabular}{|c|l|c|c|}
\hline No. & \multicolumn{1}{|c|}{ Criterio de evaluación } & SI\% & NO\% \\
\hline 1 & ¿El esquema organizacional es altamente jerarquizado? & 97.5 & 2.5 \\
\hline 2 & $\begin{array}{l}\text { ¿Existen rígidas líneas de autoridad y establecimiento formal de autorizaciones en los } \\
\text { distintos niveles de la organización? }\end{array}$ & 92.5 & 7.5 \\
\hline 3 & $\begin{array}{l}\text { ¿Existen en la organización más de dos niveles verticales de decisión; distintas a la } \\
\text { máxima autoridad, (Dirección, Gerencia y presidencia)? }\end{array}$ & 100 & 0 \\
\hline 4 & $\begin{array}{l}\text { ¿Es común que dos o más dependencias horizontales participan en la ejecución o } \\
\text { elaboración de un producto o servicio? }\end{array}$ & 25 & 75 \\
\hline 5 & $\begin{array}{l}\text { ¿Cada dependencia tiene autonomía para dar respuestas a las solicitudes de los } \\
\text { usuarios? }\end{array}$ & 0 & 100 \\
\hline 6 & ¿Las decisiones se concentran en la alta dirección? & 5 \\
\hline 7 & ¿La estructura de la organización define claramente las responsabilidades? & 95 \\
\hline 8 & $\begin{array}{l}\text { ¿Están establecidos por la alta dirección los mecanismos que garanticen la coordinación } \\
\text { de las áreas para el logro de los objetivos? }\end{array}$ & 85 & 57.5 \\
\hline 9 & $\begin{array}{l}\text { ¿La Dirección General concerta con los responsables, las actividades específicas y la } \\
\text { formulación de objetivos, metas y niveles de productividad y desempeño? }\end{array}$ & 42.5 \\
\hline 10 & ¿La entidad asume de manera ágil y oportuna los cambios internos y externos? & 50 & 50 \\
\hline 11 & $\begin{array}{l}\text { ¿La estructura establecida permite la conformación de equipos de trabajo, orientados al } \\
\text { logro de los objetivos corporativos? }\end{array}$ & 27.5 & 72.5 \\
\hline 12 & $\begin{array}{l}\text { ¿La movilidad de los funcionarios esta soportada mediante estudios técnicos de perfiles } \\
\text { y competencias? }\end{array}$ & 30 & 70 \\
\hline 13 & $\begin{array}{l}\text { ¿Se promociona la autonomía personal y de grupo para el ejercicio de las funciones, } \\
\text { planes, programas, proyectos? }\end{array}$ & 7.5 & 92.5 \\
\hline 14 & ¿La estructura organizacional está basada en la gestión de procesos? & 0 \\
\hline
\end{tabular}


Dentro de los resultados del diagnóstico a través de la aplicación de la encuesta (Tabla 2), se pueden evidenciar los siguientes problemas relacionados con la estructura organizacional y el enfoque de gestión: (i) La CGR tiene una estructura organizacional altamente jerarquizada; (ii) En todos los niveles de la CGR se evidencia la existencia de rígidas líneas de autoridad; (iii) Debido a la existencia de niveles de decisión verticales, la toma de estas es lenta; (iv) Es difícil encontrar que dos o más dependencias trabajen en la ejecución de un mismo proceso; (v) No se cuenta con un enfoque de gestión que les permita a los funcionarios ser proactivos en su trabajo; (vi) Existe una concentración de toma de decisiones en la alta dirección y las dependencias no cuentan con autonomía para dar respuesta a las solicitudes de las partes interesadas; (vii) La información fluye de manera vertical, lo que ocasiona que sea lenta y poco clara; y (viii) Se evidencia poca articulación de los grupos de trabajo intra e inter dependencias.

Teniendo en cuenta los resultados de las entrevistas y la encuesta, se considera pertinente especificar un dominio de arquitectura empresarial, armonizando la simulación de procesos con la metodología ADM, para dimensionar de manera apropiada las necesidades de la organización y así mejorar el desempeño del macro proceso de control fiscal micro.

\section{METODOLOGÍA}

El desarrollo metodológico se presenta en dos etapas: en una se describe el marco de referencia seleccionado; y en otra etapa la aplicación del modelado y simulación.

\section{Etapa 1. Marco de referencia seleccionado}

Para la especificación de los dominios de arquitectura empresarial existen diferentes marcos de referencia, los cuales según Arango y Otros (2010), determinan en qué términos se estructura dicha arquitectura. Para el estudio se seleccionó el marco de referencia TOGAF (The Open Group Architecture Framework), ya que éste cumplía con las características necesarias para el desarrollo metodológico, como las que menciona Santiago (2014):

Framework libre: El framework es de uso gratuito para instituciones sin ánimo de lucro lo cual facilita el acceso al mismo y su uso en el proyecto.

Documentación y guías de apoyo: Además de la especificación del framework para la construcción de la AE, cuenta con documentación, artefactos y guías adicionales que facilitan la construcción de los productos de cada fase definida en la metodología ADM.

Reconocimiento internacional: TOGAF es uno de los frameworks de mayor reconocimiento mundial como apoyo al desarrollo de la arquitectura empresarial de una organización.

Framework neutro e integrable: TOGAF ha sido desarrollado por el Open Group, por lo cual lo hace un framework neutro respecto a herramientas y tecnologías a utilizar y está diseñado de tal manera que permite una fácil integración con otros framework de AE y otras metodologías.

Tomando como base el marco de referencia de arquitectura empresarial TOGAF Versión 9.1 (2011) y según la metodología ADM, se identificaron las siguientes dimensiones para definir y limitar el alcance de la actividad de arquitectura, como se muestra en la tabla 3.

Tabla 3. Dimensiones para limitar el alcance

\begin{tabular}{|l|l|}
\hline Dimensión & Consideraciones \\
\hline Amplitud & $\begin{array}{l}\text { ¿Cuál es la extensión total de la empresa, y con qué parte de } \\
\text { esa extensión debería tratar el esfuerzo de arquitectura? }\end{array}$ \\
\hline Profundidad & $\begin{array}{l}\text { ¿Qué nivel de detalle debería alcanzar el esfuerzo de } \\
\text { arquitectura? }\end{array}$ \\
\hline Dominios de arquitectura & $\begin{array}{l}\text { ¿Cuál o cuáles dominios de arquitectura se van a } \\
\text { especificar? }\end{array}$ \\
\hline
\end{tabular}

\section{Amplitud}

En cuanto a la extensión de la CGR para la actividad de arquitectura y debido a su tamaño, solo se tuvo en cuenta el macro proceso misional de control fiscal micro, tabla 4. 
Tabla 4. Descripción general del macro proceso de control fiscal micro

\begin{tabular}{|c|c|}
\hline \multicolumn{2}{|c|}{ Macro Proceso De Control Fiscal Micro } \\
\hline \multicolumn{2}{|c|}{ Objetivo } \\
\hline \multicolumn{2}{|c|}{$\begin{array}{l}\text { Vigilar la gestión fiscal y el control de resultados de las entidades y/o particulares que manejen fondos o bienes } \\
\text { públicos. }\end{array}$} \\
\hline Recursos & Normatividad \\
\hline $\begin{array}{l}\text { Talento humano, recursos financieros, infraestructura } \\
\text { de telecomunicaciones y sistemas de información. }\end{array}$ & $\begin{array}{l}\text { Entidades a vigilar (según resolución vigente), AUDITE, } \\
\text { Decreto } 267 \text { de } 2000 \text {, Ley } 610 \text { de } 2001 \text {, Ley } 42 \text { de } 1993 \text { y } \\
\text { Ley } 617 \text { de } 2000 .\end{array}$ \\
\hline \multicolumn{2}{|c|}{ Dependencias responsables } \\
\hline \multicolumn{2}{|c|}{$\begin{array}{l}\text { Contralorías Delegadas y Direcciones de Vigilancia Fiscal de los sectores: medio ambiente, agropecuario, } \\
\text { infraestructura, defensa justicia y seguridad, minas y energía, social, gestión pública y la Unidad de Investigaciones } \\
\text { Especiales para la Corrupción. }\end{array}$} \\
\hline \multicolumn{2}{|c|}{ Procesos relacionados con el macro proceso } \\
\hline
\end{tabular}

\section{Profundidad}

Para tal fin se aplicó el primer paso del ciclo de vida bajo el enfoque BPM (Business Process Model), fig. 1; con el fin de entender los procesos de negocio de la CGR, dando como resultado que un punto neurálgico y del cual dependen el rumbo y los resultados del proceso auditor, es la fase de planeación. Dicha fase tiene como objetivo definir el alcance y la estrategia de auditoría a partir del conocimiento del auditado y de la evaluación de los controles para mitigar los riesgos, fig. 2.

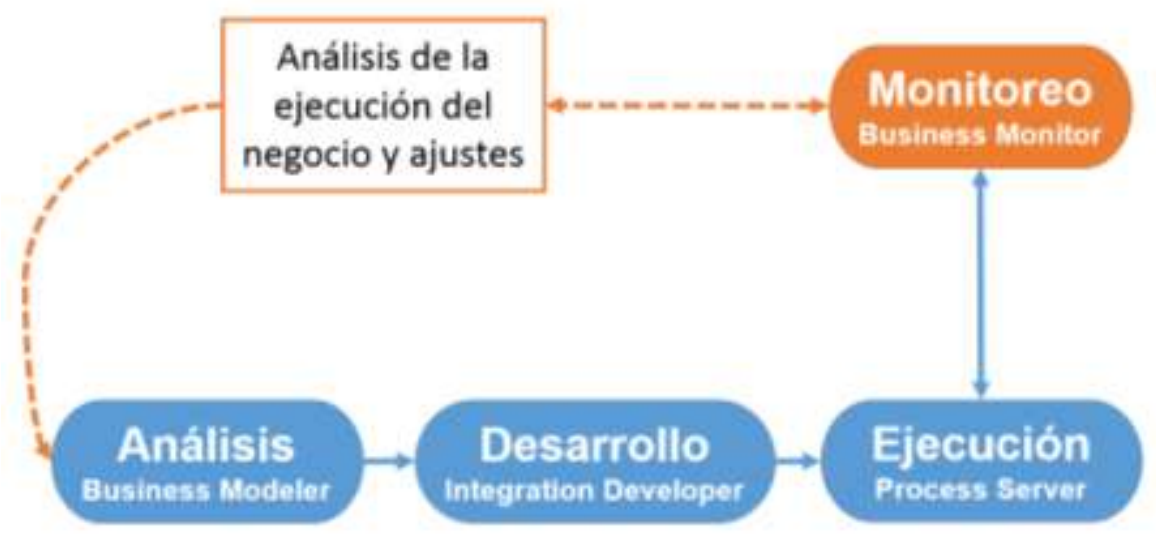

Fig. 1. Ciclo de vida bajo un enfoque BPM (Fuente: www.ibm.com)

\section{Dominios de arquitectura}

Teniendo en cuenta que el método de desarrollo de la arquitectura ADM es modular, lo que admite particionar las arquitecturas dentro de una misma empresa o áreas de ésta por dominios, fue elegido el dominio de arquitectura de negocio, para lo cual se debe seleccionar una herramienta que permita definir un modelo e identificar las acciones que permitan mejorar su desempeño.

De tal modo, la definición del proceso para el modelamiento y simulación se inició haciendo uso del dominio de arquitectura de negocio a nivel de diagnóstico, el cual implica realizar un análisis de la situación actual y un inventario de procesos de negocio. Adicional a lo anterior, en la publicación CIO del Ministerio de TI de Colombia (2013), se menciona que este diagnóstico debe incluir un análisis de integración, interoperabilidad e integración entre procesos y subprocesos. 


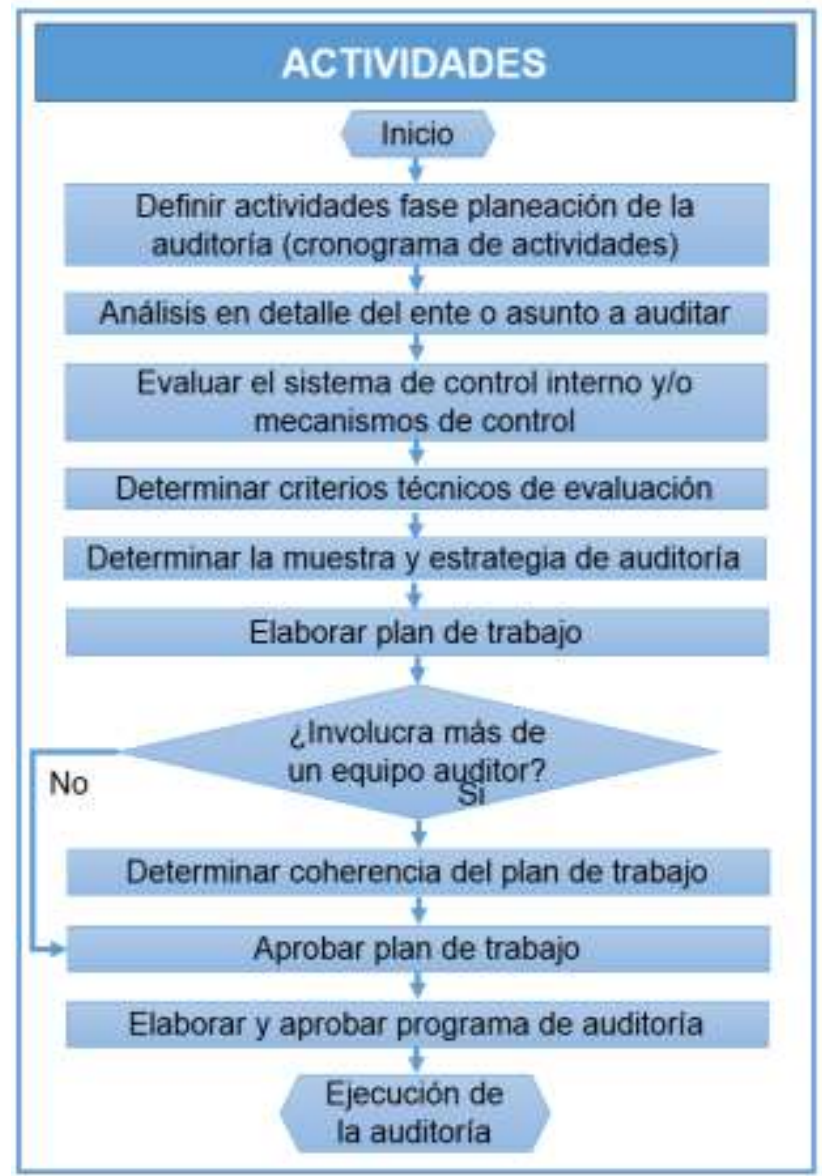

Fig. 2. Fase de planeación de la auditoría

\section{Etapa 2. Aplicación del modelado y simulación a través de la herramienta}

Con base en las actividades descritas para la fase de planeación del proceso auditor en la guía de auditoría de la CGR, se realizó el modelo de procesos sobre el cual se ejecutara la simulación, fig. 3. En éste modelo se pueden identificar las siguientes actividades y subprocesos: inicia con la notificación del memorando de asignación y comprende la elaboración del cronograma de actividades para la misma; el análisis en detalle del ente o asunto a auditar; la evaluación del sistema de control interno o de los mecanismos de control; la determinación de los criterios técnicos de evaluación, alcance y estrategia de auditoría, hasta la elaboración y aprobación del plan de trabajo y de los programas de auditoría.

La modelación se realizó haciendo uso del software Bizagi Process Modeler, el cual es una herramienta que permite modelar y documentar procesos de negocio bajo el estándar Business Process Model and Notation (BPMN). Dicha herramienta fue elegida porque cumplía con los siguientes requerimientos: i) Capacidad de hacer pruebas que simulen la ejecución de procesos para mejorar el desempeño y solucionar problemas; ii) Capacidades gráficas para las pruebas; iii) Capacidad de obtener archivos de un origen de datos externo; y iv) Capacidad de representar el proceso mediante herramienta visual y disponibilidad de lenguaje de programación para personalizar al máximo las simulaciones.

La mencionada herramienta usa el estándar BPSim (Business Process Simulation), corriendo los cuatro niveles, i) Validación; ii) Análisis de tiempo; iii) Análisis de recursos; iv) Análisis de calendarios. En cada nivel de la vista de simulación se añade mayor grado de detalle al modelo, la posibilidad de crear escenarios para distintas combinaciones de datos y analizar sus resultados.

\section{Validación del proceso}

Este se realiza con el fin de evaluar la estructura y la lógica del diagrama desarrollado, se programaron las compuertas de decisión con las probabilidades correspondientes a cada uno de los flujos de proceso. Así por ejemplo, la compuerta "Determinar la estrategia de auditoría" tiene un $95 \%$ de posibilidades que el flujo de trabajo vaya por el "Enfoque en controles" y el $5 \%$ que sea "Enfoque sustantivo" fig, 4. Estos porcentajes se obtuvieron de distribuciones históricas de auditorías realizadas en anteriores PGA (Plan General de Auditoría de la CGR) bajo variadas condiciones. 


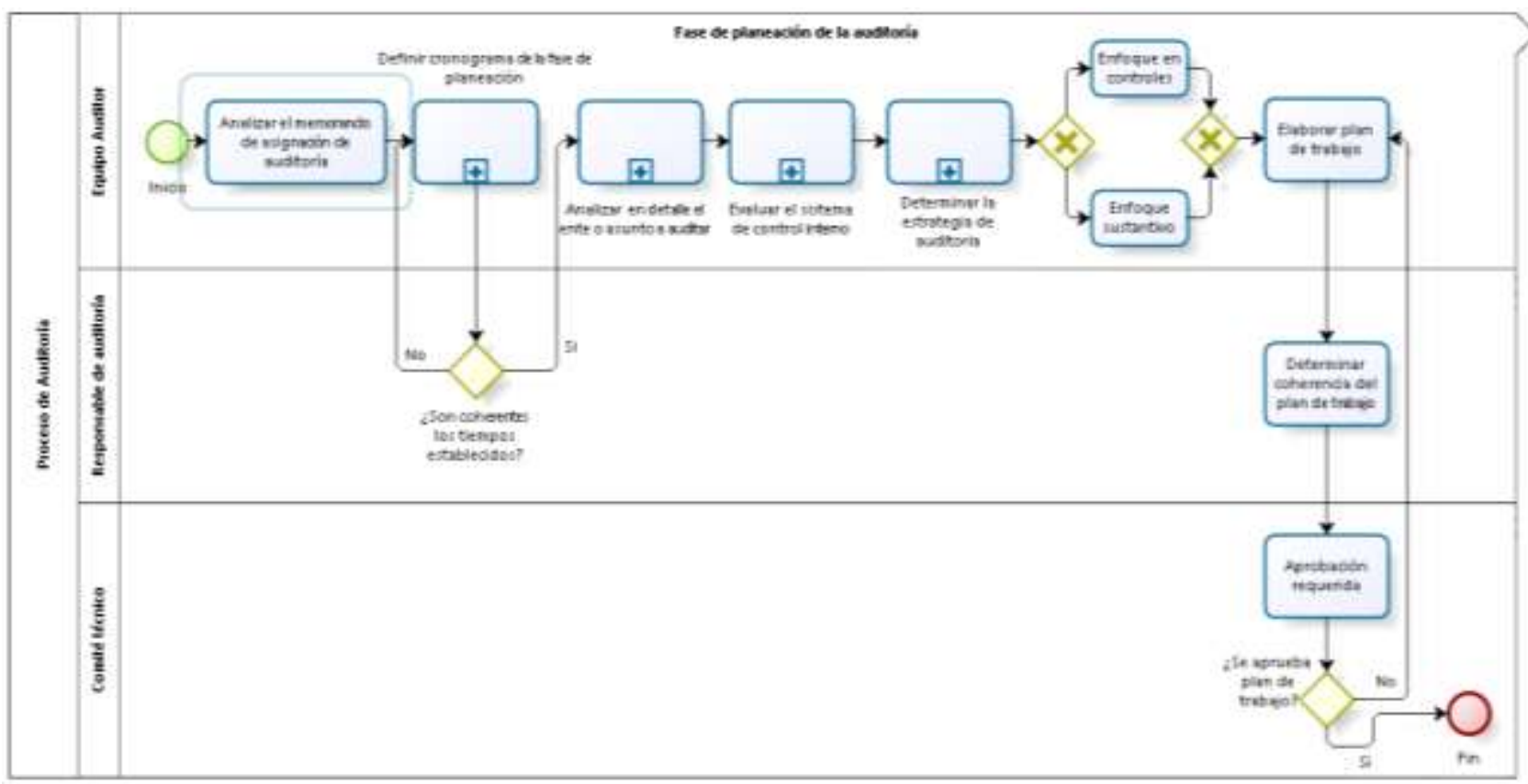

Fig. 3. Modelo de la Fase de planeación de la auditoría en formato BPMN 2.0.

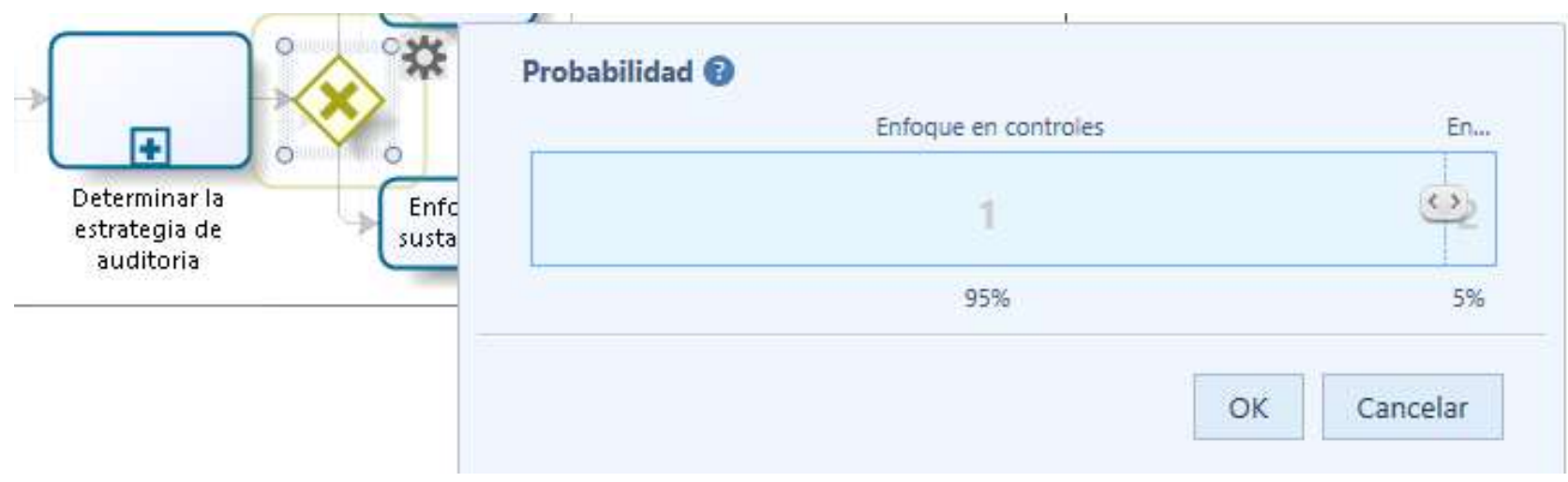

Fig. 4. Validación del modelo a través de compuertas

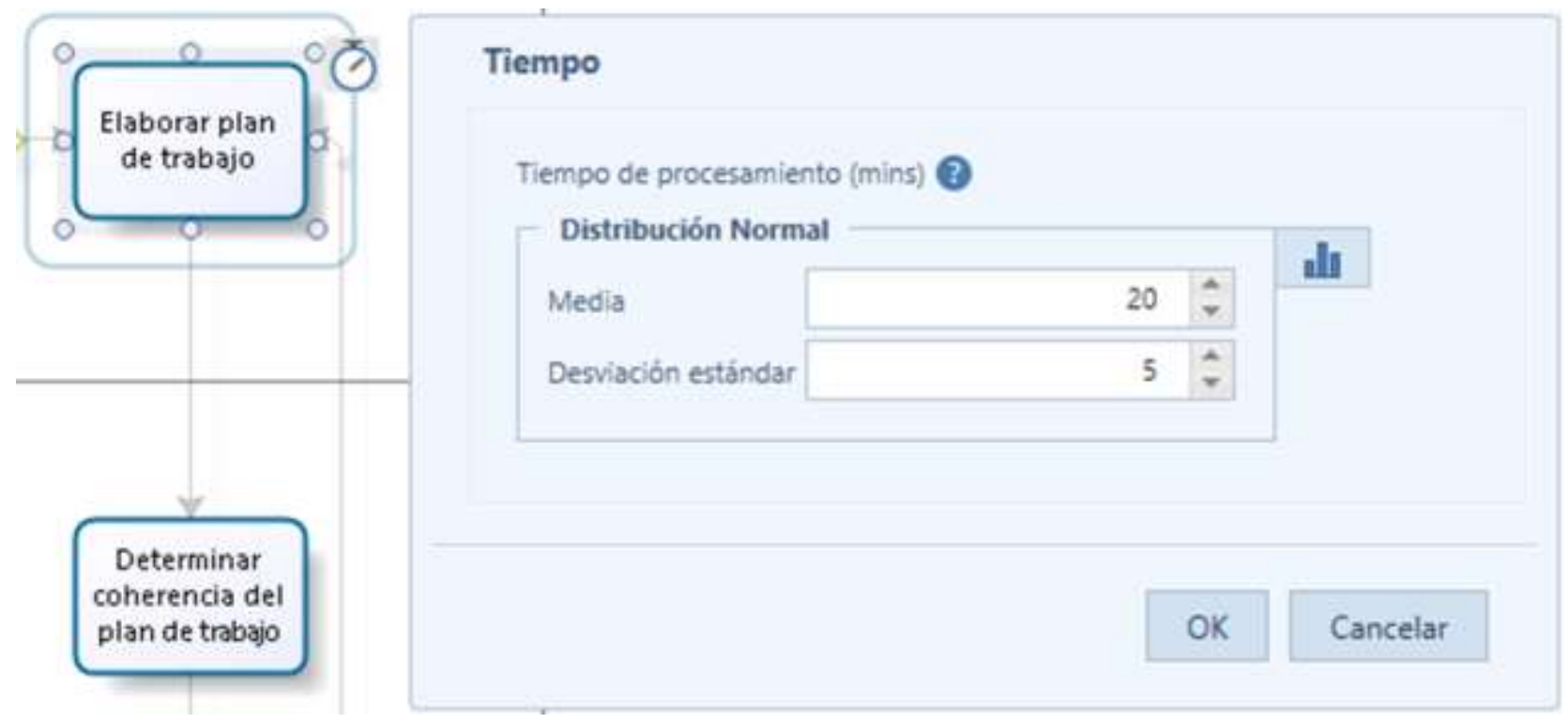

Fig. 5. Nivel de análisis de tiempo 


\section{Análisis de tiempo}

Este nivel se simula para comprender el tiempo de procesamiento de cada actividad y el intervalo de tiempo entre la generación de las instancias del proceso. Los resultados deben mostrar el desempeño del proceso de planeación de auditoría, consolidando los tiempos mínimos, máximos, medios y totales de procesamiento, para lo cual se establece la duración de cada tarea y subproceso asignándole valores determinísticos o probabilísticos a los tiempos, según ocurre en la realidad. Por ejemplo, el tiempo del subproceso "Elaborar plan de trabajo" se distribuye probabilísticamente mediante una distribución normal, fig. 5.

\section{Análisis de recursos}

Adicional a los datos introducidos en los niveles anteriores, en este nivel se incluyen los recursos disponibles, tales como costo y personal. Por ejemplo, al subproceso de "Evaluar el sistema de control interno" se le realiza la asignación de 2 auditores. fig. 6.

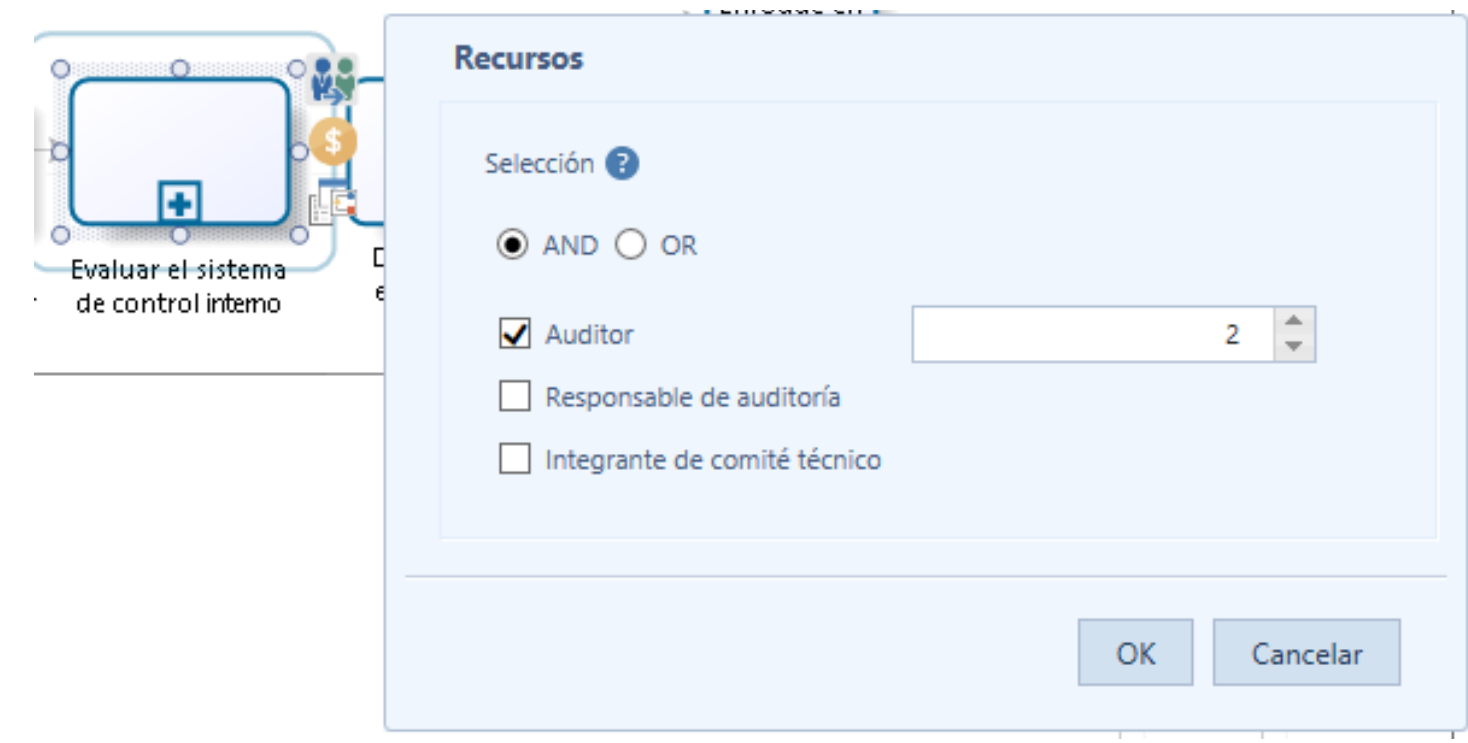

Fig. 6. Nivel de análisis de recursos

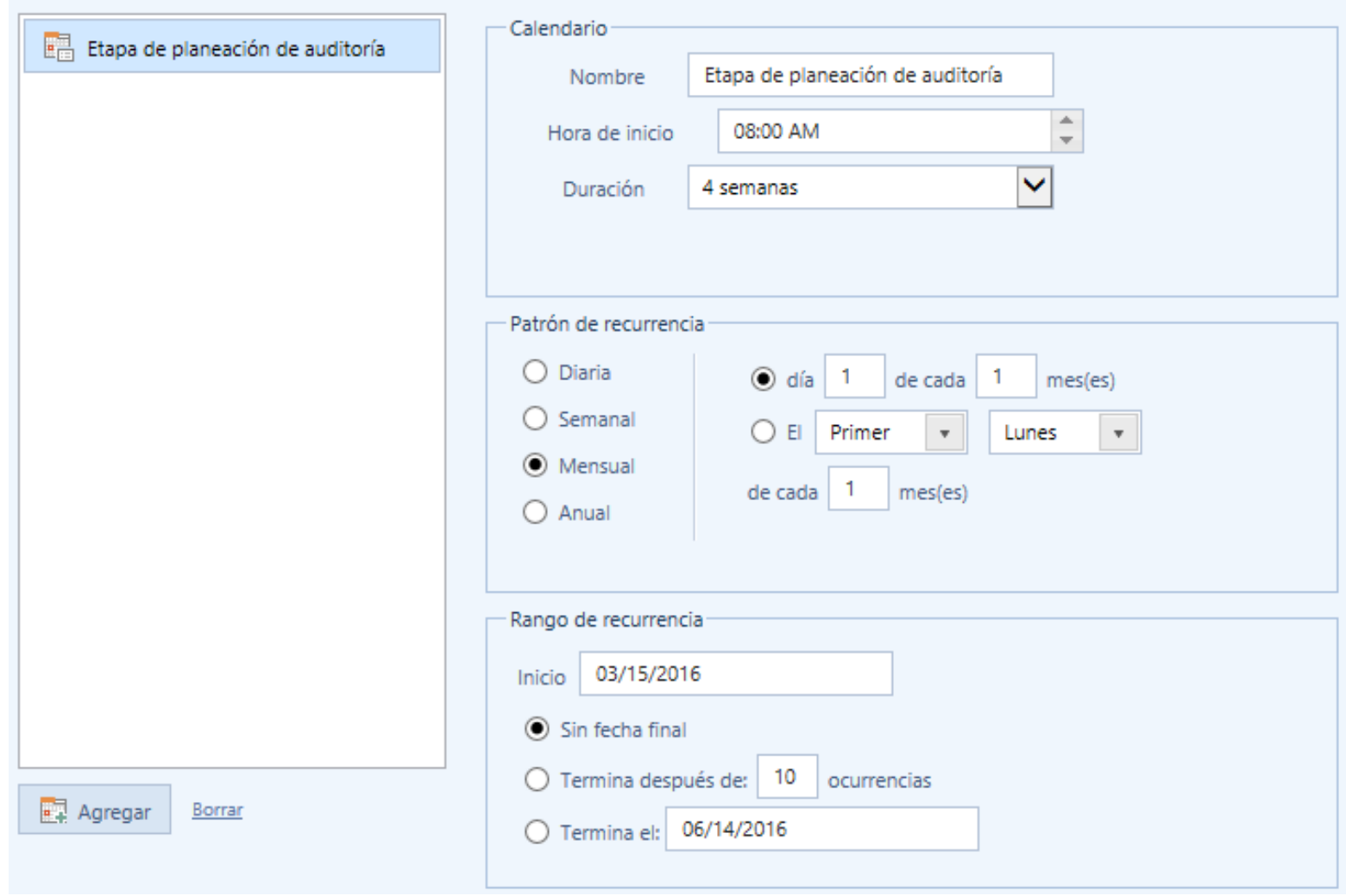

Fig. 7. Nivel de análisis de calendarios 


\section{Análisis de Calendarios}

En cuanto a este nivel, se obtiene como resultado que la disponibilidad de tiempo por parte de los auditores, en diferentes etapas del proceso es exagerada, lo que se debe a la espera de aprobaciones por parte de instancias superiores, impidiendo que el flujo continúe hasta que se obtenga una respuesta positiva. En este nivel se definen los calendarios de los procesos en periodos de tiempo, que para este caso corresponde a la duración de la etapa de planeación del proceso auditor con su patrón y rango de recurrencia, fig. 7.

\section{RESULTADOS Y DISCUSIÓN}

Al armonizar la simulación de procesos con la metodología ADM, a nivel de diagnóstico del dominio de arquitectura de negocio, se ha logrado la descripción de los procesos, sus actores y su organización lógica, dando como resultado que un punto neurálgico y del cual dependen el rumbo y los resultados del proceso auditor, es la fase de planeación, a la cual se le aplicó el modelado y la simulación corriendo los cuatro niveles del estándar BPSim (Business Process Simulation).

En el primer nivel del proceso de simulación, se muestra que se activan todos los caminos del proceso y se asegura que todas las instancias creadas en realidad fueron finalizadas. Además se evalúa el número de instancias que pasan a través de cada flujo de secuencia, actividad y evento final. Encontrándose en éste nivel, que la tarea "aprobación requerida" genera un alto número de instancias, lo que repercute en el subproceso de "Elaborar plan de trabajo" hasta que éste se valide y apruebe.

Al ejecutar el nivel de análisis de tiempo, simulando 10 instancias correspondientes al mismo número de auditorías, se obtiene como resultado tabla 5, la duración de cada una de las tareas, así como el tiempo total en que estas estuvieron en funcionamiento. Como se puede evidenciar en estos resultados, los tiempos no son coherentes con los trabajos desarrollados, lo que se puede justificar por la rigidez del proceso al depender de la aprobación del responsable de auditoría y del comité técnico, generando un cuello de botella en los flujos de trabajo, lo que puede en un momento determinado entorpecer el inicio de las otras fases del proceso auditor, como la de ejecución y hasta el mismo informe de auditoría.

Tabla 5. Resultados de simulación - Validación de proceso y análisis de tiempo

\begin{tabular}{|c|c|c|c|c|}
\hline Nombre & Tipo & $\begin{array}{l}\text { Instancias } \\
\text { completadas }\end{array}$ & $\begin{array}{l}\text { Instancias } \\
\text { iniciadas }\end{array}$ & $\begin{array}{l}\text { Tiempo } \\
\text { total (d) }\end{array}$ \\
\hline Proceso de Auditoría & Proceso & 10 & 10 & 28.42 \\
\hline $\begin{array}{l}\text { Analizar el memorando de asignación de } \\
\text { auditoría }\end{array}$ & Tarea & 10 & 10 & 1 \\
\hline Elaborar plan de trabajo & Tarea & 23 & 23 & 5 \\
\hline Determinar coherencia del plan de trabajo & Tarea & 23 & 23 & 5 \\
\hline ¿Se aprueba plan de trabajo? & Compuerta & 23 & 23 & \\
\hline Aprobación requerida & Tarea & 23 & 23 & 5 \\
\hline $\begin{array}{l}\text { ¿Son coherentes los tiempos } \\
\text { establecidos? }\end{array}$ & Compuerta & 16 & 16 & \\
\hline Compuerta exclusiva & Compuerta & 10 & 10 & \\
\hline Enfoque en controles & Tarea & 6 & 6 & 1 \\
\hline Enfoque sustantivo & Tarea & 4 & 4 & 0 \\
\hline Compuerta exclusiva & Compuerta & 10 & 10 & \\
\hline Fin & Evento de Fin & 10 & & \\
\hline Inicio & Evento de inicio & 10 & & \\
\hline $\begin{array}{l}\text { Definir cronograma de la fase de } \\
\text { planeación }\end{array}$ & Proceso & 16 & 16 & 0.4 \\
\hline $\begin{array}{l}\text { Analizar en detalle el ente o asunto a } \\
\text { auditar }\end{array}$ & Proceso & 10 & 10 & 5 \\
\hline Evaluar el sistema de control interno & Proceso & 10 & 10 & 3 \\
\hline Determinar la estrategia de auditoria & Proceso & 10 & 10 & 3 \\
\hline
\end{tabular}


Como consecuencia de lo anterior se crearon dos escenarios del modelo, para analizar el comportamiento al realizar cambios en el proceso, el primer escenario es configurado con datos que se obtuvieron de distribuciones históricas de auditorías anteriores y un segundo escenario, en el que se eliminó el reproceso "Aprobación requerida" el cual generaba un cuello de botella en los flujos de trabajo al depender de dos instancias, asignándole la aprobación al responsable de auditoría, cuando estas se presentan de manera repetitiva por falta de coherencia en su planteamiento, observándose una reducción del $28.8 \%$, en el tiempo total del proceso, tabla 6.

Tabla 6. Resumen análisis de simulación de escenarios

\begin{tabular}{|l|l|r|r|r|}
\hline Nombre & Escenario & \multicolumn{1}{|l|}{$\begin{array}{l}\text { Instancias } \\
\text { completadas }\end{array}$} & $\begin{array}{l}\text { Instancias } \\
\text { iniciadas }\end{array}$ & $\begin{array}{l}\text { Tiempo } \\
\text { total (d) }\end{array}$ \\
\hline Proceso de Auditoría & Escenario 1 & 10 & 10 & 28.4 \\
\hline Proceso de Auditoría & Escenario 2 & 10 & 10 & 20.2 \\
\hline Elaborar plan de trabajo & Escenario 1 & 23 & 23 & 5 \\
\hline Elaborar plan de trabajo & Escenario 2 & 12 & 12 & 3 \\
\hline Determinar coherencia del plan de trabajo & Escenario 1 & 23 & 23 & 5 \\
\hline Determinar coherencia del plan de trabajo & Escenario 2 & 12 & 12 & 3 \\
\hline ¿Se aprueba plan de trabajo? & Escenario 1 & 23 & 23 & - \\
\hline ¿Se aprueba plan de trabajo? & Escenario 2 & 12 & 12 & - \\
\hline Aprobación requerida & Escenario 1 & 23 & 23 & 5 \\
\hline Aprobación requerida & Escenario 2 & 10 & 10 & 0.8 \\
\hline
\end{tabular}

Al analizar la simulación de los dos escenarios, se puede comprobar una correspondencia con los resultados de las entrevistas y de la encuesta en por lo menos lo siguiente: (i) Debido a la existencia de niveles de decisión verticales, la toma de estas es lenta; (ii) No se cuenta con un enfoque de gestión que les permita a los funcionarios ser proactivos en su trabajo; (iii) Existe una concentración de toma de decisiones en la alta dirección y las dependencias no cuentan con autonomía para dar respuesta a las solicitudes de las partes interesadas; y (iv) La información fluye de manera vertical, lo que ocasiona que sea lenta y poco clara.

En cuanto al nivel de recursos, al ejecutar la simulación los valores varían constantemente, pero se deja denotar que tanto el responsable de auditoría como el comité técnico son los que tienen el más alto grado de uso. Para el nivel de calendarios, se obtiene como resultado que la disponibilidad de tiempo por parte de los auditores, en diferentes etapas del proceso es exagerada, lo que se debe a la espera de aprobaciones por parte de instancias superiores, impidiendo que el flujo continúe hasta que se obtenga una respuesta positiva.

Por otro lado y partiendo de la idea que las organizaciones están estructuradas de acuerdo con sus flujos de trabajo y las interrelaciones entre los actores involucrados, al hacer un análisis de los resultados del modelado y simulación en la fase de planeación del proceso auditor en la CGR, se puede identificar claramente que su estructura basa su estilo de dirección en una rigurosa estandarización, donde la especificación de un dominio de arquitectura de negocio, es fundamental como soporte para el análisis del estado actual de la entidad con miras al aumento del rendimiento de sus procesos como lo menciona Ortega y Otros (2012).

Por lo anterior, la CGR se puede enmarcar dentro de los parámetros de las organizaciones que requieren el uso de herramientas que le permitan entender sus procesos y especificar los dominios de la arquitectura empresarial.

\section{CONCLUSIONES}

De los resultados expuestos en el punto anterior se pueden exponer las siguientes conclusiones:

Especificar un dominio de arquitectura empresarial, armonizando la simulación de procesos con la metodología $A D M$, en uno de los macro procesos de la CGR, permite dimensionar de una manera más apropiada las necesidades de entendimiento de la organización para mejorar el desempeño de sus procesos de negocio. 
Como una contribución de la presente investigación, se utilizó una herramienta de modelado y simulación basada en el estándar BPSim (Business Process Simulation), la cual a partir de un enfoque de BPM, permitió detallar las fases del ciclo ADM representando las condiciones estructurales de la organización y evaluando el desempeño con diferentes configuraciones, mostrando los cuellos de botella y la sub y sobre utilización de recursos en un diagrama de flujo, utilizando símbolos y formas.

Finalmente, se considera pertinente iniciar nuevas investigaciones donde no solamente se modele y simule la fase de planeación, sino otras como la ejecución e informe del proceso auditor como complemento a la construcción de un prototipo de arquitectura empresarial de los procesos misionales de la CGR.

\section{AGRADECIMIENTOS}

Los autores agradecen a la Universidad Nacional de Colombia sede Manizales y a la Contraloría General de la República de Colombia, por el apoyo institucional y financiero al desarrollo de esta investigación, la cual hace parte del proyecto de tesis doctoral "Incidencia de la administración con enfoque burocrático en los resultados del control fiscal micro, caso aplicado a la Contraloría General de la República de Colombia".

\section{REFERENCIAS}

AGR - Auditoría General de la República, La impostergable reingeniería del control fiscal territorial, (en línea), 2013. http://www.auditoria.gov.co/index.php/bibliotecavirtual/prensa/doc_details/1081-laimpostergable-reingenieria-del-control-fiscalterritorial?tmpl=component. Acceso: 6 de diciembre (2015)

Arango, M. D., Londoño, J. E. y Zapata, J. A., Arquitectura empresarial - una visión general, http://revistas.udem.edu.co/index.php/ingenierias/article/view/46, ISSN: 1692-3324, Rev. Ingenierías, 9(16), $101-111(2010)$

Bizagi, Bizagi BPM Suite Functional Description, (en línea), 2014.

https://www.bizagi.com/docs/BizAgi\%20Functional\%20Description.pdf. Acceso: 18 de diciembre (2015)

Bolaños, S. J. y López, S. A., LMPS como propuesta alterna a BPMN para el modelado de procesos de software, http://www.scielo.org.co/scielo.php?script=sci_arttext\&pid=S0123921X2012000400012\&Ing=en\&tIng=., ISSN: 0123-921X, Rev. Tecnura, 16(34), 157-170 (2012)

CGR - Contraloría General de la República, Control Fiscal Participativo, Economía Colombiana, edición 315 enero-febrero (2011)

$\mathrm{CIO}$ - Ministerio de TI de Colombia, Arquitectura empresarial, (en línea), 2013. http://www.mintic.gov.co/gestionti/615/articles-5322_Revista_pdf.pdf. Acceso: 18 de febrero (2015)

De Velasco, J. A., Gestión por procesos, 4ª edición, 45-60, ESIC Editorial, Madrid, España, (2010)

Geyer, R. W. y Fourie, C. J., Determining the suitability of a business process modelling technique for a particular application, http://www.scielo.org.za/scielo.php?script=sci_arttext\&pid=S222478902015000100019\&Ing=en\&nrm=iso, ISSN: 1012-277X, Rev. South African Journal of Industrial Engineering, 26(1), 252-266 (2015)

González, S. E., La constitución colombiana y las funciones gerenciales del sector público, http://www.redalyc.org/articulo.oa?id=265419724005, ISSN: 1900-3803, Rev. Entramado, 5(2), 64-79 (2009)

OMG - Object Management Group, Business Process Model and Notation (BPMN), (en línea), 2011. http://www.omg.org/spec/BPMN/2.0/. Acceso: 22 de mayo (2015)

Ortega, D., Uzcategui, E. y Guevara, M., EAIF: Un framework de arquitectura empresarial orientado a servicio en correspondencia con MDA, http://www.scielo.org.ve/scielo.php?script=sci_arttext\&pid=S131648212012000100003\&Ing=es\&tIng=es., ISSN: 1316-4821, Rev. Universidad Ciencia y Tecnología, 16(62), 23-32 (2012)

Santiago, C. P., Propuesta de Arquitectura Empresarial para una universidad como un apoyo a su desarrollo frente a los retos del Siglo XXI, Twelfth LACCEI Latin American and Caribbean Conference for Engineering and Technology, 1-11, Guayaquil, Ecuador, 22 a 24 de julio (2014). 
The Open Group, Togaf 9.1. - Gruía (Latin American Spanish), (en línea), 2011. https://www2.opengroup.org/ogsys/ServePublicationGraphic?publicationid=13158. Acceso: 18 de agosto (2015).

Villanueva, J., La simulación de procesos, clave en la toma de decisiones,

http://www.revistadyna.com/busqueda/la-simulacion-de-procesos-clave-en-toma-de-decisiones, Rev. Dyna Ingeniería e Industria, 83(4), 221-227 (2008)

WfMC - Workflow Management Coalition, Process Definition Interface, (en línea), 2005. http://www.xpdl.org/standards/xpdl-2.0/TC-1025_xpdl_2_2005-10-03.pdf. Acceso: 8 de agosto (2015) 